\title{
Enabling Critical Analytic Thinking
}

\author{
Richard May \\ Pacific Northwest National Laboratory \\ richard.may@pnl.gov
}

\author{
Jörn Kohlhammer \\ Fraunhofer IGD \\ joern.kohlhammer@igd.fraunhofer.de
}

\author{
David S. Ebert \\ Purdue University \\ ebertd@purdue.edu
}

\begin{abstract}
Motivation - To introduce the audience to visual analytics and its relevance to cognitive and decision sciences by stimulating dialog on the current state of visual analytics research, unresolved challenges and future directions. Research approach -Current research will be presented and demonstrated in the areas of public health and safety. Findings/Design - The panelists will introduce the audience to visual analytics, its relationship to the digital information explosion, and promote discussions on challenges faced by visual analytics relative to NDM methodologies. Research limitations/Implications - Visual analytics is still an emerging science that is continuing to evolve. The implication of visual analytics is a new approach to analyzing large dynamic heterogeneous information spaces resulting in faster and better decisions. Originality/Value - Visual analytics represents a new science to support analytical decision making. Take away message - The expanding digital universe is confounding many analysis tasks with vast amounts of diverse information. Visual analytics is an emerging science to address these challenges through supporting an interactive, human-driven decision making process.
\end{abstract}

Keywords

Visual analytics, analysis, interactive.

\section{INTRODUCTION}

Visual Analytics is defined as the science of analytic reasoning facilitated by interactive visual interfaces (Thomas \& Cook, 2005). This relatively new approach is built on fundamentals in scientific and information visualization but has a much broader science foundation, including cognitive and decision sciences, knowledge sciences, scalable mathematical and statistical sciences, and thereby enables critical analytic thinking. This panel will address defining visual analytics, the current state of the science from research centers around the world, technologies deployed today, and many application examples.

When we create a mental picture, speak of the mind's eye, say "I see" to indicate understanding, or use many other vision-based metaphors, we are expressing the innate connection among vision, visualization, and our reasoning processes. This science of analytical reasoning provides the reasoning framework upon which one can build both strategic and tactical visual analytics technologies for a broad range of problem spaces. Analytical reasoning is central to the task of applying human judgments to reach conclusions from a combination of evidence and assumptions.

Many challenges still need to be addressed by the emerging science of visual analytics. A new human information discourse for discovery has to be developed. One that embodies a new interaction paradigm based around cognitive aspects of critical thinking. New visual paradigms better equipped to deal with previously unheard of scale, heterogeneity, and dynamic streaming temporal data flows have to be created. Other challenges include information representations, capturing analytical insight, knowledge dissemination, and many others.

\section{KEY TOPICS}

Visual analytics strives to facilitate the analytical reasoning process by creating software that maximizes human capacity to perceive, understand, and reason about complex and dynamic data and situations. It must build upon an understanding of the reasoning process, as well as an understanding of underlying cognitive and perceptual principles, to provide task-appropriate interactions that allow practitioners to have a true discourse with their information. The goal is to facilitate high-quality human judgment with a limited investment of the practitioner's time.

The science of analytical reasoning underpins the research areas presented on this panel. It provides a basis and a direction for the science of visual representations and interactions and forms a foundation for the principles of depicting information in meaningful and novel visual representations. Public health and public safety are two of the application areas with visual analytics applications being deployed today and will be explored by the panelists. 
PUBLIC HEALTH: The role of public health surveillance is to collect, analyze and interpret data about biological agents, diseases, risk factors, and other health events in order to provide timely dissemination of collected information to decision makers. Unfortunately, data sources vary widely in accuracy and reliability, and frequently, unusual health trends, such as outbreaks or poisonings, have an incidence profile (signal) that is obscured by the statistical noise. To help investigators overcome these issues, a suite of statistical and visual analytical tools that couple novel data sources with human health data has been developed. The primary data sources include emergency room data, general visits of pets to pet hospitals, State Board of Animal Health data, and data from public health news feeds, such as ProMed. These analytical tools and datasets are brought together in a visual analytics environment. The application provides investigators with the ability to visually search the animal and human health data for clusters in both a statistical model view and a spatio-temporal view. By providing linked graphical and statistical analysis views for public health officials, we are improving event detection and response, while reducing false positives.

PUBLIC SAFETY: Law enforcement investigators need to detect patterns, trends, and entities of interest in streaming incident data. The large amount of heterogeneous information available through federated law enforcement query services, however, makes this difficult. Investigators also need the ability to collect new information in the field, such as interviews, photographs, and video, and the ability to share this information instantly with colleagues for incident investigation. To address these needs, we are transitioning advanced web-based and mobile incident analysis and collaboration technologies to front-line law enforcement and counter-terrorism personnel at several regional law enforcement agencies around the United States. We have developed a suite of analytic capabilities including identification of temporal, spatial, and topical incident patterns and threat trends. The analytical toolkit offers novel location-based services and watch-and-warn techniques for access to key information conducive to decision-making and collaboration in the field. The system is optimized for tactical, daily use with anytime, anywhere access to shared data and analytic resources. Currently, users query for data by supplying a name or other identifier. But when these searches return many results, it can be difficult to determine if a suspect has outstanding warrants or if there are attributes - like a common vehicle description - that connect multiple crimes, especially when running a query from the field. The toolkit addresses this problem. With clustering and analysis tools deployed via web browsers and PDAs, search results from many different data providers can be integrated and presented in visual groups that reveal their hidden structure.

\section{ACKNOWLEDGMENTS}

Development of material for this session was supported by the National Visualization and Analytics Center (NVAC), a U.S. Department of Homeland Security Program operated by the Pacific Northwest National Laboratory, a U.S. Department of Energy Office of Science laboratory and by Fraunhofer IGD and Purdue University.

\section{REFERENCES}

Thomas, J \& Cook, K. (2005) Illuminating the Path: The Research and Development Agenda for Visual Analytics, Los Alamitos, CA: IEEE Press, pp. 200. 